\title{
Designing an Efficient Hybrid Optical Cavity
}

\author{
Thomas Siday $^{1}$, Robert J Thompson ${ }^{1,2}$, Samuel Glass ${ }^{1}$, Ting-Shan Luk ${ }^{3,4}$, John L Reno ${ }^{3,4}$, Igal Brener ${ }^{3,4}$ \\ and Oleg Mitrofanov ${ }^{1,3}$ \\ ${ }^{1}$ Electronic and Electrical Engineering, University College London, London, UK \\ ${ }^{2}$ London Center for Nanotechnology, University College London, London, WC1H 0AH \\ ${ }^{3}$ Center for Integrated Technologies, Sandia National Laboratory, Albuquerque, NM 87185, USA \\ ${ }^{4}$ Sandia National Laboratory, Albuquerque, NM 87185, USA
}

\begin{abstract}
We present an efficient terahertz (THz) detector based on an optically thin hybrid cavity. We use experimental and numerical methods to design efficient detectors, finding a hybrid cavity structure with a photoconductive (PC) layer as thin as $50 \mathrm{~nm}$ which absorbs almost $80 \%$ of light at the operation wavelength. These optically thin detectors are well suited to near-field microscopy and terahertz component integration.
\end{abstract}

\section{INTRODUCTION}

$\mathrm{T}$ he performance of photoconductive $\mathrm{THz}$ detectors based on low temperature grown (LT) GaAs is limited primarily by the properties of the bulk semiconductor material. While the sub-picosecond switching time of LT-GaAs allows for THz detection, the relatively long absorption length when compared to the mean free path of photo-excited charge carriers limits detection performance, as the majority of photo-excited carriers recombine before being collected at an electrode. The use of plasmonic nanostructured electrodes has been shown to increase the proportion of collected carriers [1]; however, the narrow spacing of the electrodes may lead to increased dark current.

Alternatively, photonic structures trap light within a thin photoconductive layer, effectively reducing the optical absorption length. The mean free path of photo-excited carriers remains constant, resulting in increased carrier collection. A scheme for trapping light within a thin PC layer was recently proposed for application to $\mathrm{THz}$ detectors using a hybrid cavity design [2]. The structure is shown in Figure 1(a). A molecular beam epitaxy (MBE) grown LT-GaAs PC layer is situated between a Distributed Bragg Reflector (DBR), consisting of 5 pairs of alternating $\mathrm{Al}_{0.55} \mathrm{Ga}_{0.45} \mathrm{As}$ and GaAs layers, and an array of lithographically defined gold nano-antennas, electrically isolated from the PC layer with a thin $(15 \mathrm{~nm})$ layer of aluminum oxide.

Here, we use finite-difference time-domain (FDTD) simulations [3] to design efficient hybrid cavity structures. We provide insight into the dependence on nano-antenna length, array periodicity and PC layer thickness. Fabricated hybrid cavity structures are integrated into $\mathrm{THz}$ PC detectors and device performance is compared to identical detectors without the nano-antenna array.

\section{RESULTS}

Figure 1(b, c) shows respectively, numerical and experimental reflectivity and transmission of the hybrid cavity. Without the nano-antenna array, the device acts as a DBR (dashed lines), with high reflectivity and low transmission within the stopband. When the nano-antenna array is introduced (solid lines) the reflectivity is suppressed within the stopband to a minimum of $\sim 5 \%$ at the design wavelength $(\sim 810 \mathrm{~nm})$, while transmission through the device remains low. This suppression in reflectivity indicates a high absorption resonant mode within the hybrid cavity.

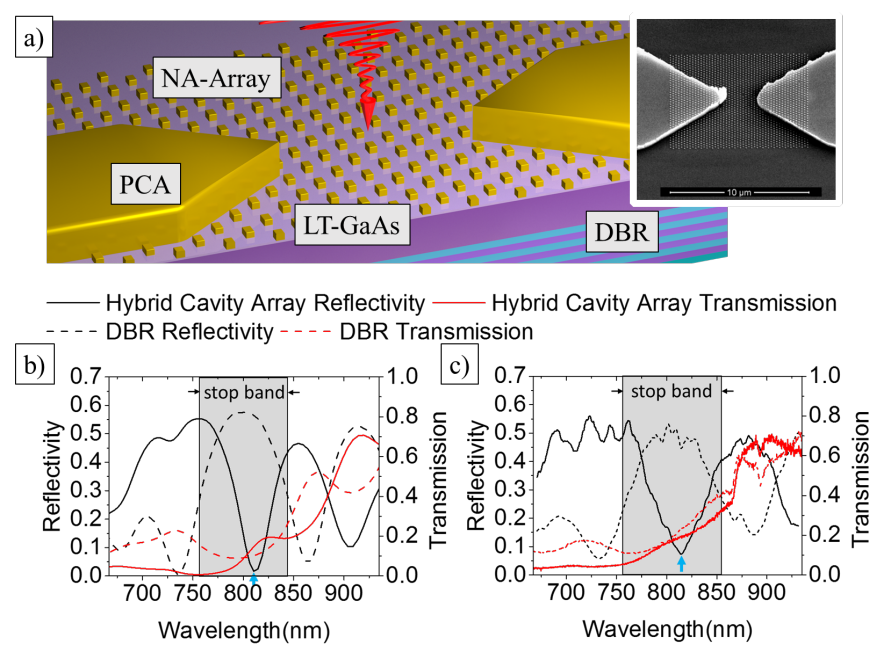

Fig. 1. (a) Visualization of the hybrid cavity structure. An SEM of the device is inset. (b) Numerical simulations and (c) experimental measurements of the reflectivity and transmission spectra of the hybrid cavity structure, with (solid lines) and without (dashed lines) the nano-antenna array [1, 4].

By varying the thickness of the LT-GaAs PC layer, periodic maxima occur at the design wavelength for thicknesses $50 \mathrm{~nm}$, $160 \mathrm{~nm}$, and $270 \mathrm{~nm}$ (Fig. 2). The location of these maxima can be approximated analytically with a k-vector condition incorporating the PC layer and DBR:

$$
k=\frac{m_{r} \pi}{L_{G a A s} n_{G a A s}+m_{D B R} L_{D B R} n_{D B R}}
$$

Here, $L_{G a A s}$ is the thickness of the PC layer with refractive index $n_{\text {GaAs. }} m_{D B R}$ is the number of DBR pairs with thickness $L_{D B R}$ and average refractive index $n_{D B R} . m_{r}$ is a half-integer, and represents the resonance mode order of the cavity. Within the DBR stopband (Fig. 1(b)), the k-vector condition agrees with the numerical simulations for $m_{r}=5.5,6.5$, and 7.5 (Fig. 2). Optimal PC layer thickness can therefore be expressed as $L_{G a A s}=\left(\frac{1}{4}+\frac{1}{2} N\right) \lambda / n_{\text {GaAs }}$ where $N=0,1,2, \ldots$ 


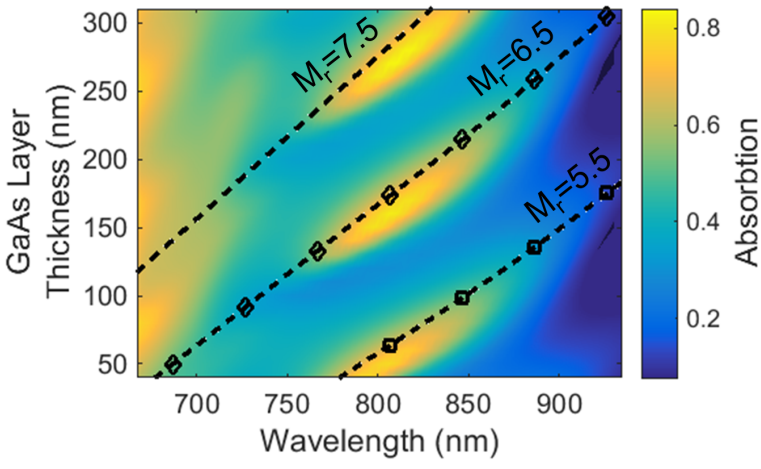

Fig. 2. Normalized absorption (defined as 1 - (Reflection + Transmission)) for increasing GaAs layer thickness. The dashed lines show the k-vector condition (1) for mode orders $m_{r}=5.5,6.5$ and 7.5 [4].

FDTD simulations predict the length of the nano-antennas affects both the wavelength at which reflections are suppressed, and the efficiency of the suppression (Fig. 3(a)). For experimental confirmation, nano-antenna arrays with different electron beam exposures are fabricated (Fig. 3(b)). Varying the electron beam exposure affects both length and width of the nanoantennas, however only the long axis, parallel to the polarization of the incident light, is critical for device performance.

The wavelength dependence can be explained intuitively by considering the phase of the reflected light. Reflections from the DBR have approximately constant phase across the DBR stopband, whereas the phase of light reflected from the nanoantennas shifts significantly, across the nano-antenna resonance. Reflection is suppressed when the above components interfere destructively. For increasing nano-antenna length, their resonant wavelength decreases, resulting in the dependence seen in Figure 1(c).

By using the optimal antenna length $(90 \mathrm{~nm})$, thee candidate hybrid cavity designs can be identified with optical absorption $79 \%, 82 \%$ and $83 \%$ for PC layer thicknesses $50 \mathrm{~nm}, 160 \mathrm{~nm}$ and $270 \mathrm{~nm}$ respectively. The nano-antenna array periodicity was found not to significantly affect hybrid cavity absorption [4] and so was nominally set to $340 \times 250 \mathrm{~nm}$.
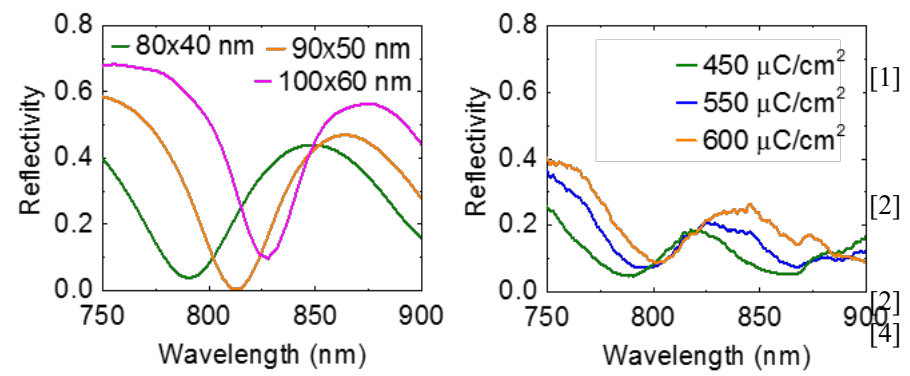

Fig. 3. (a) Numerical and (b) Experimental reflectivity spectra for increasing nanoantenna size [4].

A $270 \mathrm{~nm}$ hybrid cavity structure was integrated into a photoconductive $\mathrm{THz}$ detector, and compared to a similar detector without the nano-antenna array with $\mathrm{THz}$ time domain spectroscopy (TDS). Figure 4 shows the peak detected THz field. The peak at $\lambda=815 \mathrm{~nm}$ corresponds to the hybrid cavity mode. Inset is the detected THz waveforms at this wavelength.

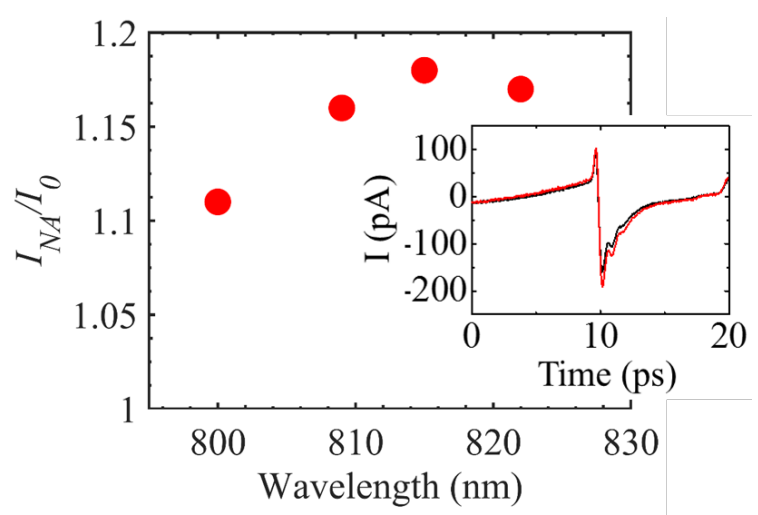

Fig. 4. Enhancement in detector sensitivity at different wavelengths. Inset: $\mathrm{THz}$ waveforms detected with the nano-antenna array (red line) and without (black line) [4].

The sensitivity enhancement measured using the THz TDS system is not as great as the predictions of the numerical simulations and optical characterization. This is due to significant optical absorption within the DBR, and the nano-antenna array, absorption in which does not contribute to the detected THz signal. Absorption within the DBR can be almost entirely eliminated by replacing the GaAs with low $\mathrm{Al}$ content AlGaAs. It is also possible to reduce the nano-antenna absorption by either using a metal with lower loss than gold at the operation wavelength, or by reducing the density of nanoantennas in the array.

\section{SUMMARY}

In this study, we identify and optimize the various parameters required to develop an efficient $\mathrm{THz}$ detector based on an optical hybrid cavity. Several designs are identified, including a structure with a $50 \mathrm{~nm}$ PC layer which absorbs almost $80 \%$ of incident light. However, the significant absorption within the nano-antenna array and DBR limit device performance when used for $\mathrm{THz}$ detection. A device design with reduced parasitic absorption is expected to have significantly increased detector performance.

\section{REFERENCES}

[1] C. W. Berry, N. Wang, M. R. Hashemi, M. Unlu, and M. Jarrahi, 'Significant performance enhancement in photoconductive terahertz optoelectronics by incorporating plasmonic contact electrodes.', Nat. Commun., vol. 4, p. 1622, 2013.

[2] O. Mitrofanov, I. Brener, T. S. Luk, and J. L. Reno, 'Photoconductive Terahertz Near-Field Detector with a Hybrid Nanoantenna Array Cavity', ACS Photonics, vol. 2, no. 12, pp. 1763-1768, Dec. 2015.

[3] Lumerical Solutions Inc., http://www.lumerical.com/tcad-products/fdtd/.

[4] R. J. Thompson et al., 'Optically thin hybrid cavity for terahertz photoconductive detectors', Appl. Phys. Lett., vol. 110, no. 4, p. 41105, Jan. 2017. 